\title{
Increased expression of Notch 1 in a rat liver transplantation model
}

\author{
LIHONG CHEN ${ }^{1,2 *}$,WUBING HE ${ }^{3 *}$, KAIDA HUANG $^{1}$, LIN ZHENG $^{1}$, \\ LINGYUN GAO $^{1}$, JINGFENG LIU ${ }^{2,4}$ and AIMIN HUANG ${ }^{1}$
}

\footnotetext{
${ }^{1}$ Department of Pathology, School of Basic Medical Sciences of Fujian Medical University, Fuzhou, Fujian 350004;

${ }^{2}$ Department of Pathology, Mengchao Hepatobiliary Hospital of Fujian Medical University, Fuzhou, Fujian 350025;

${ }^{3}$ Provincial Clinical Medical College, Fujian Provincial Hospital, Fujian Medical University, Fuzhou, Fujian 350001;

${ }^{4}$ Divison of Hepatobiliary Surgery, Hepatic Disease Center, The First Affiliated Hospital of Fujian Medical University, Fuzhou, Fujian 350005, P.R. China
}

Received September 3, 2014; Accepted June 11, 2015

DOI: $10.3892 / \mathrm{mmr} .2015 .4026$

\begin{abstract}
Liver transplantation is the standard treatment for end-stage liver failure; however, rejection can result in allograft failure. In order to investigate the role of Notch 1 during rejection, the present study evaluated Notch 1 expression, as well as the levels of immune reactivity, in rat liver allografts. A heterotopic liver transplantation model was established using Dark Agouti (DA) rats as donors and Lewis rats as recipients (DA/Lewis), with DA recipient rats serving as controls (DA/DA). The concentration levels of immune reactivity markers and serum Notch 1 were measured on days 3, 5, and 7. The overall survival was significantly shorter ( $<10$ days) in the DA/Lewis group, as compared with the DA/DA group $(\mathrm{P}<0.0001)$. The concentration levels of serum alanine aminotransferase and total bilirubin were significantly higher 5 and 7 days following transplantation in the DA/Lewis group, as compared with the DA/DA group $(\mathrm{P}<0.001)$. The concentration levels of serum Notch 1 were significantly higher in the DA/Lewis group, as compared with the DA/DA group on days 3,5 , and 7 following transplantation $(\mathrm{P}<0.0001)$. These results indicate that the expression levels of serum Notch 1 significantly increase during liver allograft rejection, suggesting that Notch 1 is involved in the mechanism underlying liver allograft rejection. Notch 1 may serve as a marker of acute rejection in a rat liver transplantation model.
\end{abstract}

Correspondence to: Professor Aimin Huang, Department of Pathology, School of Basic Medical Sciences of Fujian Medical University, 1 Xue Yuan Road, Fuzhou, Fujian 350004, P.R. China E-mail: draimin@163.com

${ }^{*}$ Contributed equally

Key words: Notch 1, acute rejection, liver transplantation

\section{Introduction}

Liver transplantation is the standard treatment for patients with end-stage liver failure. Despite immunosuppressive drugs providing excellent short-term outcomes following transplantation, acute and chronic rejection episodes still occur and affect long-term graft function. Non-invasive monitoring of graft-specific immune activation would allow for the early diagnosis, and ultimately, the prediction and management of acute or chronic liver rejection (1). Therefore, novel methods are required in order to accurately predict acute rejection following transplantation.

The Notch gene, originally identified in Drosophila (2), is highly conserved in both invertebrates and vertebrates (3). The Notch signaling pathway consists of four Notch receptors (Notch 1-4), and five structurally similar Notch ligands (Delta-like 1, 3, and 4, and Jagged 1 and 2) (4,5). Notch signaling has an important role in the regulation of cell fate, and influences the growth and survival of progenitor cells (6). The Notch signaling pathway regulates various aspects of embryonic development, as well as differentiation processes and tissue homeostasis in numerous adult organ systems (7).

A recent study demonstrated that Notch receptors and ligands are highly expressed in the central and peripheral immune systems (7). The Notch signaling pathway has an important role in regulating the differentiation, proliferation, and function of mature lymphocytes, including peripheral $\mathrm{T}$ cells. Once naïve $\mathrm{T}$ cells migrate to the periphery in response to antigens, in the presence of appropriate signals the naïve $\mathrm{T}$ cells become activated and exert their function (7). Notch receptor expression has been linked to $\mathrm{T}$ cell activation, proliferation, and cytokine production (7), suggesting that these signals may be closely associated with the immune reaction to allografts. Following renal transplantation, Notch 1 expression was associated with the immune state of recipients, indicating that the expression levels of Notch 1 may predict long-term renal function. Notch 1 may therefore serve as a marker of acute rejection and long-term renal function following renal transplantation (8).

Peripheral blood is easily accessible and may be used to assess biomarkers that accurately reflect or predict immune 
Table I. Detection of the concentration levels of serum Notch 1 in the liver transplant recipients, as determined by ELISA ( $\mu$ mol/l).

\begin{tabular}{lccc}
\hline Group & Day 3 & Day 5 & Day 7 \\
\hline DA/DA & $9.313 \pm 1.011$ & $9.030 \pm 0.742$ & $8.791 \pm 0.721$ \\
DA/Lewis & $13.405 \pm 0.802^{\mathrm{a}}$ & $17.527 \pm 0.824^{\mathrm{a}}$ & $23.605 \pm 0.731^{\mathrm{b}}$ \\
\hline
\end{tabular}

Concentration levels of serum Notch 1 were significantly higher in the Dark Agouti (DA)/Lewis group, as compared with the DA/DA group on days 3, 5 and 7 ( ${ }^{\mathrm{P}}<0.0001$ vs. DA/DA rats), as determined by an unpaired two-tailed Student's t-test. In addition, these concentrations significantly increased over time in the $\mathrm{DA} /$ Lewis group ( ${ }^{\mathrm{P}} \mathrm{P}<0.0001 \mathrm{vs}$. DA/DA rats).

responses to a graft. The present study established a rat liver transplantation model in order to examine whether increased expression levels of Notch 1 in the peripheral blood were predictive of early acute immune rejection.

\section{Materials and methods}

Animals. Male Dark Agouti (DA) and Lewis rats (age, 8-10 weeks old; weight, $230 \pm 20 \mathrm{~g}$ ) were purchased from the Laboratory Animal Center of the Second Affiliated Hospital at Harbin Medical University (Heilongjiang, China), and from the Shanghai SLAC Laboratory Animal Co. Ltd. (Shanghai, China), respectively. All rats were housed in microisolator cages in the barrier facility of the Fujian Medical University (Fuzhou, China). The rats were housed at $27^{\circ} \mathrm{C}$ in $45 \%$ humidity with $12 \mathrm{~h}$ light/dark cycle, with ad libitum access to food and water. All experiments were approved by the Ethics Committee of Fujian Medical University (Fuzhou, China).

Establishment of a rat heterotopic liver transplantation model. A heterotopic liver transplantation model was established using DA rats $(n=50)$ as donors and Lewis rats $(n=25)$ as recipients (DA/Lewis), with DA rats (DA/DA) $(n=25)$ serving as recipients in the control group (9-11). Five rats from each group were sacrificed on days 3, 5, and 7 post-transplantation, prior to liver tissue sample harvesting. The overall allograft survival rates were monitored in 10 rats from each group, with allograft rejection being histologically confirmed.

Liver function measurements. The serum concentration levels of total bilirubin (TBIL) and alanine transaminase (ALT) were measured using the caffeine method and rate method according to the manufacturer's instructions (Cobas 8000 Biochemical Analyzer; Roche Diagnostics, Basel, Switzerland) on days 3, 5, and 7 following liver transplantation.

Notch 1 quantification by ELISA. The concentration levels of serum Notch 1 were quantified on days 3,5, and 7 following liver transplantation by ELISA (450 nm) using Quantikine M kits (Yueyan Biotech, Shanghai, China), according to the manufacturer's instructions. The Bio-Rad 550 ELISA Plate-Reader was used (Bio-Rad Laboratories, Inc., Hercules, CA, USA).

Histological analysis. Allografts were histologically examined following fixing of allograft tissues in $4 \%$ paraformaldehyde and embedding in paraffin (Sinopharm Chemical Reagent Co., Shanghai, China). on days 3,5, and 7 , and the overall rejection rates were assessed according to the Banff schema (12). Paraffin sections $(5 \mu \mathrm{m})$ were cut, then dewaxed and rehydrated through reducing graded alcohols, using a standard protocol: Three changes of xylene (Sinopharm Chemical Reagent Co.), 3 min each; two changes of $100 \%$ ethanol, 2 min each; 95\% ethanol, $1 \mathrm{~min} ; 70 \%$ ethanol, $1 \mathrm{~min}$. Tissue sections were then stained with hematoxylin (Amresco, Solon, $\mathrm{OH}, \mathrm{USA}$ ) and eosin (Sinopharm Chemical Reagent Co.) for histological examination and were observed by microscopy (BX46; Olympus, Tokyo, Japan). The pathological features of acute rejection included the presence of inflammatory infiltrates in the portal tracts, bile duct damage, and endotheliitis, with at least two of these three features required for diagnosis $(13,14)$. All histological evaluations were performed in a double-blinded manner by two researchers.

Statistical analysis. SPSS 12 (SPSS, Inc., Chicago, IL, USA) analytical software was used for all statistical analyses. The results were presented as the mean \pm standard deviation, and compared by one-way analysis of variance or Student's t-test. Graft survival was analyzed by life table methods, with differences in survival assayed by the log-rank test. $\mathrm{P}<0.05$ was considered to indicate a statistically significant difference.

\section{Results}

Liver function evaluation. The concentration levels of normal serum ALT and TBIL in the Lewis rats were 26.3 $\pm 7.3 \mathrm{U} / 1$ and $11.36 \pm 4.35 \mu \mathrm{mol} / 1$, respectively (data not shown). A total of 5 and 7 days following transplantation, the concentration levels of both TBIL and ALT were significantly higher in the DA/Lewis group, as compared with the DA/DA group $(\mathrm{P}<0.001)$ (Figs. 1A and $\mathrm{B})$. These results indicate that liver function in the DA/Lewis group was continuously being restored following transplantation.

Histological assessment of donor liver grafts. The liver grafts in the DA/Lewis group exhibited moderate to severe acute rejection. Pathological changes included mixed infiltrate, infiltration of most ducts by inflammatory cells, and severe perivenular inflammation extending into the perivenular parenchyma. Conversely, the liver grafts in the DA/DA group exhibited no evidence of rejection (Fig. 2A-F).

Survival of liver allografts in recipients. Liver grafts survived $>90$ days in the DA/DA group, but $<10$ days in the DA/Lewis group $(\mathrm{P}<0.0001)$; (Fig. 3). These findings indicate severe 


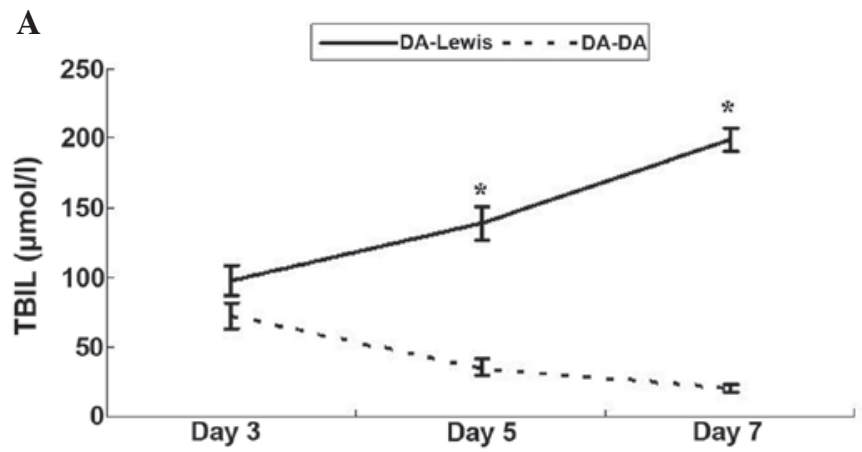

B

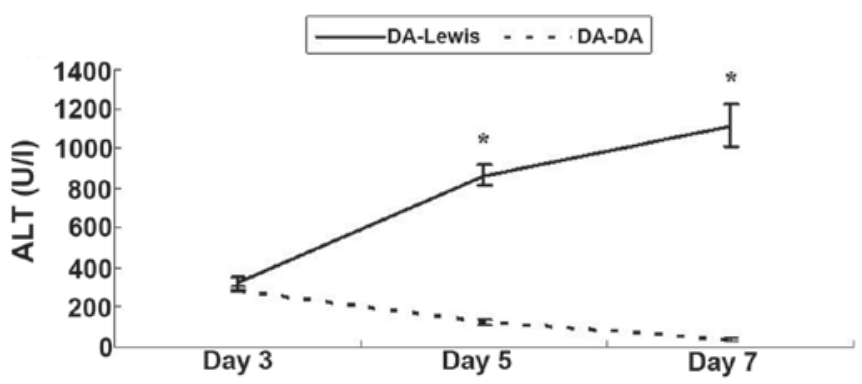

Figure 1. (A) Concentration levels of serum total bilirubin (TBIL) 3,5, and 7 days following liver transplantation in the Dark Agouti (DA)/Lewis and DA/DA groups. The concentrations levles of TBIL were significantly higher 5 and 7 days following transplantation in the DA/Lewis group, as compared with the DA/DA group ( $\mathrm{P}<0.001$ ). (B) Concentration levels of serum alanine transaminase (ALT) 3, 5, and 7 days following liver transplantation in the DA/Lewis and DA/DA groups. The concentrations levels of ALT were significantly higher 5 and 7 days following transplantation in the DA/Lewisgroup, as compared with the the DA/DA group $\left({ }^{*} \mathrm{P}<0.001\right)$.
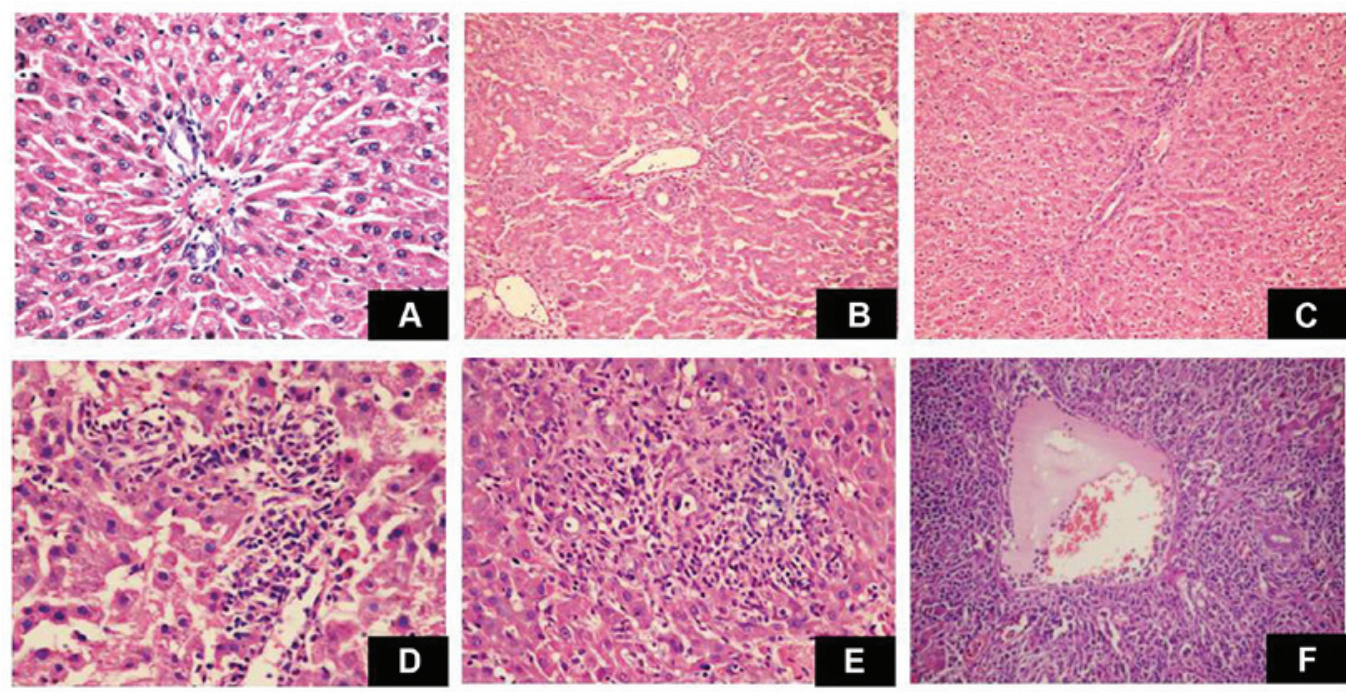

Figure 2. Histological assessment of donor liver grafts on (A and D) days 3, (B and E) 5, and (C and F) 7 following transplantation in the (A-C) Dark Agouti (DA)/DA and (D-F) DA/Lewis groups. The liver grafts in DA/Lewis group exhibited moderate to severe acute rejection, whereas the liver grafts in the DA/DA group exhibited no evidence of rejection. Magnification, $\mathrm{x} 400$; hemotoxylin and eosin staining.

acute rejection in the $\mathrm{DA} /$ Lewis rats, whereas no rejection was observed in the DA/DA group.

Serum Notch 1 levels. The concentration levels of serum Notch 1 were significantly higher in the DA/Lewis group, as compared with the DA/DA group on days 3,5 and $7(\mathrm{P}<0.0001)$ (Table I). These concentrations increased significantly over time in the DA/Lewis group $(\mathrm{P}<0.0001)$, suggesting a correlation between Notch 1 concentration and the progression of acute liver rejection.

\section{Discussion}

The present study established a rat liver transplantation model in order to examine the correlation between immune allograft rejection and Notch 1 levels in peripheral blood. The results indicated the presence of both acute rejection and increasing serum concentration levels of Notch 1 over time in the DA/Lewis group, suggesting that Notch 1 concentration may serve as a marker for early acute rejection.

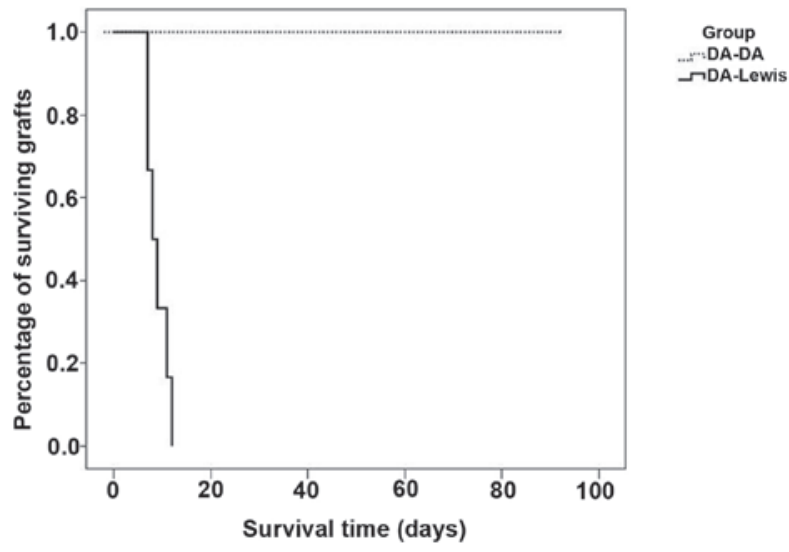

Figure 3. Overall survival in the Dark Agouti (DA)/Lewis and DA/DA groups. Overall survival was significantly shorter in the DA/Lewis group, as compared with the DA/DA group $(<10$, vs. $>90$ days, $\mathrm{P}<0.0001)$.

Non-invasive monitoring of graft-specific immune activation would allow for the early diagnosis, and ultimately, the 
prediction and pre-emptive management of acute or chronic rejection (1). Peripheral blood is easily accessible and may be used to identify and monitor biomarkers that accurately reflect, detect, or predict detrimental immune responses to grafts (1). In the present study, livers were transplanted from DA rats into either Lewis or DA rats (control), in order to evaluate liver function, histology, and survival. The results demonstrated that the concentration levels of TBIL and ALT were significantly higher in the DA/Lewis group, as compared with the DA/DA group, 5 and 7 days following transplantation $(\mathrm{P}<0.0001)$. The liver grafts in the DA/Lewis group exhibited moderate to severe acute rejection, and overall survival was significantly shorter in the DA/Lewis group, as compared with the DA/DA group $(\mathrm{P}<0.0001)$. These findings indicated that a model of acute rejection of liver transplants had been successfully established.

Notch signaling pathways have important roles in regulating the proliferation and function of mature lymphocytes $(6,7)$. Notch signaling has also been implicated as an important regulator of peripheral $\mathrm{T}$ cell activation and effector cell differentiation (15), and Notch signals may be closely associated with immune reactions in the allograft. Notch 1 expression in peripheral blood mononuclear cells (PBMCs) has previously been shown to correlate with acute rejection and long-term renal function following renal transplantation, with the expression of Notch 1 in PBMCs increasing prior to the increase in the concentration levels of serum creatinine (8). Similarly, in the present study the concentration levels of serum Notch 1 were higher in the DA/Lewis group, as compared with the DA/DA group $(\mathrm{P}<0.0001)$, and the concentration levels of Notch 1 increased significantly over time in the DA/Lewis group, as compared with the DA/DA group $(\mathrm{P}<0.0001)$.

Notch signaling has been reported to critically influence the differentiation of activated $\mathrm{T}$ cells into $\mathrm{T}$ helper 1 cells, which control cellular immunity $(15,16)$. Furthermore, Notch gene expression was induced in primary $\mathrm{CD} 4{ }^{+} \mathrm{T}$ cells following specific peptide/antigen stimulation (17). Notch activity contributes to peripheral $\mathrm{T}$ cell responses, including $\mathrm{CD}^{+}$ $\mathrm{T}$ cells, via augmentation of a positive feedback loop (17). $\mathrm{T}$ cell receptor activation of peripheral $\mathrm{T}$ cells in vitro has been shown to upregulate the expression of Notch 1 , which was correlated with increased $\mathrm{T}$ cell proliferation and interferon- $\gamma$ cytokine production (18).

Previous studies have reported that Notch signaling may induce immune tolerance. Notch signaling has been implicated in the induction of regulatory $\mathrm{T}$ cell ( $\mathrm{T}$ reg) differentiation and function (19-21). T reg cells are crucial for the negative regulation of hyperactive $\mathrm{T}$ cells and immune tolerance, via the suppression of $\mathrm{T}$ cell reactivity in peripheral tissues (22-24). Notch ligands have been reported to enhance $T$ reg cell differentiation and function in vitro $(25,26)$; however, this activity has yet to be evaluated using genetic approaches (15). The results of the present study regarding the role of Notch 1 in the acute rejection model of liver transplantation appear to differ from these previous reports. Genetic approaches are required in order to clarify the importance and mechanism underlying Notch signaling in liver transplantation.

In conclusion, the present study demonstrated that the concentration levels of serum Notch 1 are significantly increased during liver allograft rejection. These results suggested that Notch 1 is involved in the mechanisms underlying liver allograft rejection. Therefore, Notch 1 may serve as a marker of acute rejection in a rat liver transplantation model. These findings suggest that the concentration levels of serum Notch 1 may predict acute rejection in rat liver transplantation.

\section{Acknowledgements}

The present study was supported by grants from the National Natural Science Foundation of China (grant no. 81202337), the Fujian Provincial Natural Foundation (grant no. 2009D066), the Fujian Medical University Foundation (grant no. 09ZD007), and the Science and Technology Project of Fuzhou (grant no. 2013-S-125-9).

\section{References}

1. Heidt S, San Segundo D, Shankar S, Mittal S, Muthusamy AS, Friend PJ, Fuggle SV and Wood KJ: Peripheral blood sampling for the detection of allograft rejection: Biomarker identification and validation. Transplantation 92: 1-9, 2011.

2. Mohr OL: Character changes caused by mutation of an entire region of a chromosome in Drosophila. Genetics 4: 275-282, 1919.

3. Chen L, Ashraf M, Wang Y, Zhou M, Zhang J, Qin G, Rubinstein J, Weintraub NL and Tang Y: The role of notch 1 activation in cardiosphere derived cell differentiation. Stem Cells Dev 21: 2122-2129, 2012.

4. Ladi E, Nichols JT, Ge W, Miyamoto A, Yao C, Yang LT, Boulter J, Sun YE, Kintner C and Weinmaster G: The divergent DSL ligand Dll3 does not activate Notch signaling but cell autonomously attenuates signaling induced by other DSL ligands. J Cell Biol 170: 983-992, 2005.

5. Bray SJ: Notch signalling: A simple pathway becomes complex. Nat Rev Mol Cell Biol 7: 678-689, 2006.

6. Hoyne GF: Notch signaling in the immune system. J Leukoc Biol 74: 971-981, 2003.

7. Radtke F, Fasnacht $\mathrm{N}$ and Macdonald HR: Notch signaling in the immune system. Immunity 32: 14-27, 2010.

8. Zheng K, Sun X, Wu W, Yang S, Cai J and Tan J: A new index for acute rejection after renal transplant: Notch receptor-1. Exp Clin Transplant 10: 433-438, 2012.

9. Macomber CW and Shah SA: A technique of recipient portal venoplasty and cuff insertion for portal revascularization in orthotopic rat liver transplantation. J Surg Res 179: 45-46, 2013.

10. Li N, Cai CJ, Wu YR and Lu MQ: A technique of recipient portal venoplasty and cuff insertion for portal revascularization in orthotopic rat liver transplantation. J Surg Res 176: 317-320, 2012.

11. Peng Y, Gong JP, Yan LN, Li SB and Li XH: Improved two-cuff technique for orthotopic liver transplantation in rat. Hepatobiliary Pancreat Dis Int 3: 33-37, 2004.

12. Höroldt BS, Burattin M, Gunson BK, Bramhall SR, Nightingale P, Hübscher SG and Neuberger JM: Does the Banff rejection activity index predict outcome in patients with early acute cellular rejection following liver transplantation? Liver Transpl 12: 1144-1151, 2006.

13. No authors listed: Banff schema for grading liver allograft rejection: An international consensus document. Hepatology 25: 658-663, 1997.

14. Hübscher SG: Transplantation pathology. Semin Liver Dis 29: 74-90, 2009.

15. Yuan JS, Kousis PC, Suliman S, Visan I and Guidos CJ: Functions of notch signaling in the immune system: Consensus and controversies. Annu Rev Immunol 28: 343-365, 2010.

16. Amsen D, Antov A and Flavell RA: The different faces of Notch in T-helper-cell differentiation. Nat Rev Immunol 9: 116-124, 2009.

17. Adler SH, Chiffoleau E, Xu L, Dalton NM, Burg JM, Wells AD, Wolfe MS, Turka LA and Pear WS: Notch signaling augments $\mathrm{T}$ cell responsiveness by enhancing CD25 expression. J Immunol 171: 2896-2903, 2003.

18. Palaga T, Miele L, Golde TE and Osborne BA: TCR-mediated Notch signaling regulates proliferation and IFN-gamma production in peripheral T cells. J Immunol 171: 3019-3024, 2003. 
19. Hoyne GF, Dallman MJ and Lamb JR: T-cell regulation of peripheral tolerance and immunity: The potential role for Notch signalling. Immunology 100: 281-288, 2000

20. Asano N, Watanabe T, Kitani A, Fuss IJ and Strober W: Notch 1 signaling and regulatory $\mathrm{T}$ cell function. J Immunol 180: 2796-2804, 2008.

21. Ostroukhova M, Qi Z, Oriss TB, Dixon-McCarthy B, Ray P and Ray A: Treg-mediated immunosuppression involves activation of the Notch-HES1 axis by membrane-bound TGF-beta. J Clin Invest 116: 996-1004, 2006.

22. Boks MA, Kager-Groenland JR, Haasjes MS, Zwaginga JJ, van Ham SM and ten Brinke A: IL-10-generated tolerogenic dendritic cells are optimal for functional regulatory $\mathrm{T}$ cell induction - a comparative study of human clinical-applicable DC. Clin Immunol 142: 332-342, 2012.
23. Gregori S, Tomasoni D, Pacciani V, Scirpoli M, Battaglia M, Magnani CF, Hauben E and Roncarolo MG: Differentiation of type $1 \mathrm{~T}$ regulatory cells $(\mathrm{Tr} 1)$ by tolerogenic $\mathrm{DC}-10$ requires the IL-10-dependent ILT4HLA-G pathway. Blood 116: 935-944, 2010.

24. Wing K, Fehérvári Z and Sakaguchi S: Emerging possibilities in the development and function of regulatory $\mathrm{T}$ cells. Int Immunol 18: 991-1000, 2006.

25. Vigouroux S, Yvon E, Wagner HJ, Biagi E, Dotti G, Sili U, Lira C, Rooney CM and Brenner MK: Induction of antigen-specific regulatory T cells following overexpression of a Notch ligand by human B lymphocytes. J Virol 77: 10872-10880, 2003.

26. Kared H, Adle-Biassette H, Fö̈s E, Masson A, Bach JF, Chatenoud L, Schneider E and Zavala F: Jagged2-expressing hematopoietic progenitors promote regulatory $\mathrm{T}$ cell expansion in the periphery through notch signaling. Immunity 25: 823-834, 2006. 